\title{
Lead angles and emitting electron energies of Io-controlled decameter radio arcs
}

\author{
S.L.G. Hess ${ }^{\text {a,* }}$, A. Pétin ${ }^{\text {b }}$, P. Zarka ${ }^{\text {b }}$, B. Bonfond ${ }^{c}$, B. Cecconi ${ }^{\text {b }}$ \\ a LASP, University of Colorado at Boulder, USA \\ ${ }^{\mathrm{b}}$ LESIA, Observatoire de Paris, CNRS, UPMC, Université Paris Diderot, France \\ ${ }^{\mathrm{c}}$ LPAP, Université de Liège, Belgique
}

\section{A R T I C L E I N F O}

\section{Article history:}

Received 7 January 2010

Received in revised form

16 March 2010

Accepted 20 April 2010

Available online 12 May 2010

Keywords:

Jupiter-Io interaction

Jovian radio arcs

Io flux tube

Auroral electron energy

\begin{abstract}
A B S T R A C T
The Io-controlled radio arcs are emissions in the decametric radio range which appear arc shaped in the time-frequency plane. Their occurrence is controlled by Io's position, so it has been for long inferred that they are powered by the Io-Jupiter electrodynamic interaction. Their frequency ranges correspond to the electron cyclotron frequencies along the Io Flux tube, so they are expected to be generated by cyclotron maser instability (CMI). The arc shape was proposed to be a consequence of the strong anisotropy of the decametric radio emissions beaming, combined with the topology of the magnetic field in the source and the observation geometry. Recent papers succeeded at reproducing the morphologies of a few typical radio arcs by modeling in three dimensions the observation geometry, using the best available magnetic field model and a beaming angle variation consistent with a loss-cone driven CMI. In the continuation of these studies, we present here the systematic modeling of a larger number of observations of the radio arcs emitted in Jupiter's southern hemisphere (including multiple arcs or arcs exhibiting abrupt changes of shape), which permits to obtain a statistical determination of the emitting field line localization (lead angle) relative to the instantaneous Io field line, and of the emitting particle velocities or energies. Variations of these parameters relative to Io's longitude are also measured and compared to the location of the UV footprints of the Io-Jupiter interaction. It is shown that the data are better organized in a reference frame attached to the UV spot resulting from the main Alfvén wing resulting from the Io-Jupiter interaction. It is proposed that the radio arcs are related to the first reflected Alfvén wing rather than to the main one.
\end{abstract}

(c) 2010 Elsevier Ltd. All rights reserved.

\section{Introduction}

Io's orbit around Jupiter is surrounded by a dense plasma torus (Bagenal, 1994; Moncuquet et al., 2002) which is in near corotation with the planetary magnetic field. Thus Io's plasma torus moves relative to Io and generates an intense corotational electric field (Goldreich and Lynden-Bell, 1969; Saur et al., 2004), and a magnetic field perturbation (Delamere et al., 2003) which leads to the propagation of Alfvén waves carrying electric currents along the Io flux tube (IFT) (Neubauer, 1980; Saur, 2004). Since the plasma torus orbits in the centrifugal plane whereas Io orbits in the equatorial plane, their relative latitude varies with the longitude, leading to a modulation of the Io-Jupiter interaction. The interaction between Jupiter and Io leads to electron acceleration in the IFT. The electron population acquires thus a distribution unstable relative to the cyclotron-maser instability (CMI) and generates radio emissions (Wu and Lee, 1979;

\footnotetext{
* Corresponding author. Tel.: +13034926944.

E-mail address: sebastien.hess@lasp.colorado.edu (S.L.G. Hess).
}

$\mathrm{Wu}, 1985)$. Depending on the acceleration processes these emissions can appear as short timescale bursts (Ellis, 1965; Hess et al., 2007b) or as long duration arcs (Queinnec and Zarka, 1998; Hess et al., 2008b). This paper deals with the latter long duration arcs (Fig. 1).

The CMI is the mechanism thought be at the origin of most planetary auroral radio emissions (Zarka, 1998) including the Io-induced Jovian decametric emission (hereafter simply referred to as DAM). It results from the resonance between the gyration of the electrons around the magnetic field lines and a right-handed circularly polarized wave with a frequency $(\omega)$ close to the local electron cyclotron frequency $\left(\omega_{c}\right)$. The CMI generates emissions in a narrow range of beaming angles symmetric relative to the magnetic field lines (i.e. the waves are emitted along a hollow cone). The opening angle $(\theta)$ of the hollow cone relative to the magnetic field vector in the source (also called beaming angle) is large, probably up to $90^{\circ}$, even if a wide range of beaming angle values has been observed (Goldstein and Thieman, 1981a; Zarka, 1988; Queinnec and Zarka, 1998).

When their occurrence is plotted versus the Jovicentric longitude of the observer (CML, for "Central Meridian Longitude") 
a

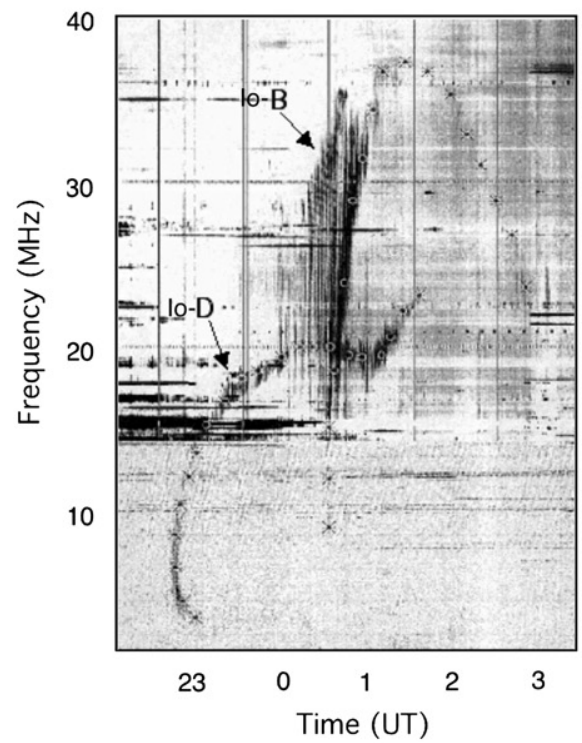

b

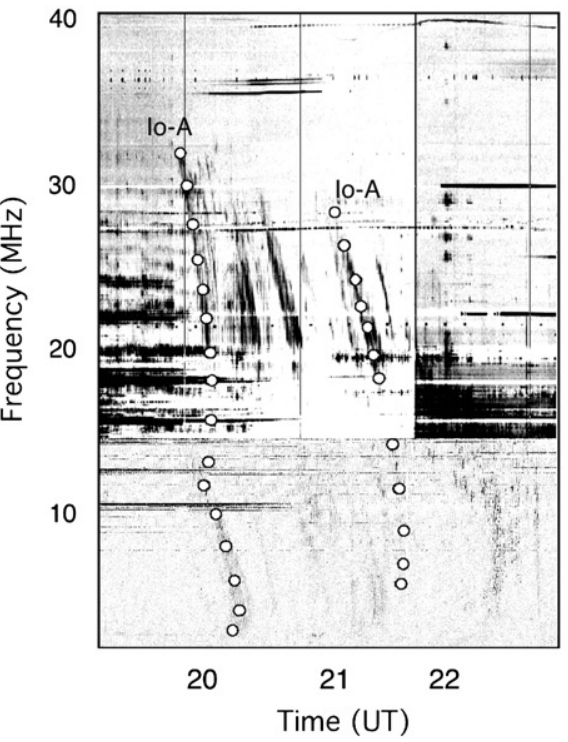

C

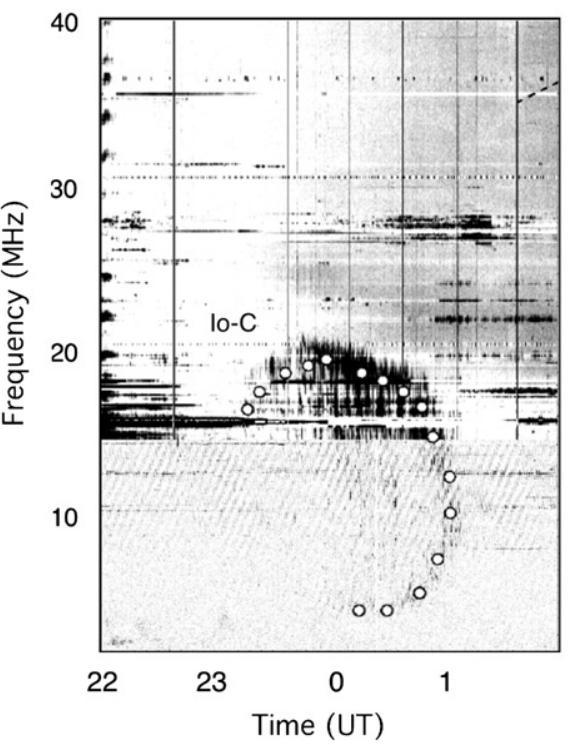

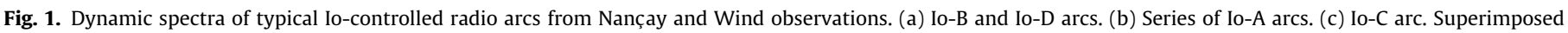
symbols along each arc follow the line of maximum intensity. Adapted from Queinnec and Zarka (1998).

and the orbital phase of Io $\left(\Phi_{I o}\right)$, Io-controlled emissions concentrate within four domains named A, B, C and D (Carr et al., 1983). These (CML, $\Phi_{I o}$ ) domains are thought to be visibility regions resulting from the variable position of the observer relative to the emission beam. Detection of the emission implies that the observer intersects the hollow cone of CMI emission. As the hollow cone opening angle is large (almost $90^{\circ}$ ), the sources are visible, in the northern (A and $\mathrm{B}$ ) and southern ( $\mathrm{C}$ and $\mathrm{D}$ ) hemispheres when Io is near the east ( $B$ and $D$ ) or west ( $A$ and $C$ ) limb of the planet (Fig. 3a). A complete description of the geometry involves a shift in longitude between Io's instantaneous magnetic field line and the emitting ("active") field line, an accurate description of the magnetic field geometry based on an internal field model, and the variation of the beaming angle versus the frequency $\theta(f)$ along the active field line. The 3D combination of these geometrical factors is responsible for the arc shape of the emissions in the time-frequency $(t-f)$ plane (Fig. 1). These shapes strongly vary from one (CML, $\left.\Phi_{I o}\right)$ domain to another, but are quite repeatable inside a given domain.

The beaming angle profile has been poorly constrained until now. Previous studies (Goldstein and Thieman, 1981a; Lecacheux et al., 1998; Queinnec and Zarka, 1998) attempted to determine it using a different methods. They all found an average beaming angle between 70 and 80. Goldstein and Thieman (1981a) and Queinnec and Zarka (1998) moreover observed decreases of the beaming angle at the highest and lowest frequencies. Lecacheux et al. (1998) proposed that the variation of the beaming angle with frequency may be due to refraction. But, no general law of variation of the beaming angle profile, that could be related to the CMI theory, was drawn from direct measurements of the beaming angle profile $\theta(f)$. Conversely, by using a beaming angle profile theoretically predicted by the loss-cone driven CMI, and modeling the visibility effects related to this anisotropic beaming, the magnetic field topology and the observation geometry, Hess et al. (2008a) succeeded at reproducing the typical shapes of a few Iocontrolled radio arcs. Ray and Hess (2008) showed that the same theoretical beaming angle profile also reproduces Voyager observations of these radio arcs, after correction of refraction effects in the source at low frequencies $(f<10 \mathrm{MHz})$.
At Earth, the auroral radio emissions generally occur along magnetic field lines connected to the auroral spots observed in the ultraviolet (UV) on the top of the planet's ionosphere (Goldstein and Thieman, 1981b). The two phenomena are thought be generated by the same populations of accelerated electrons, which for a part precipitate in the ionosphere (generating auroras) and for a part are reflected by magnetic mirroring (generating radio emissions). In the case of Io-Jupiter interaction, IR (Connerney et al., 1993) and UV (Prangé et al., 1996) auroral spots have been observed near the footprints of the modeled, unperturbed field line connecting Io to Jupiter, with a longitudinal difference of few degrees called "lead angle". These footprint signatures are generally composed of several spots (see Fig. 2a and Gérard et al., 2006; Bonfond et al., 2008). UV observations have led to classify these spots as: (i) the main Alfvén wing spot (MAW) which has in average the lowest lead angle and marks the position of the Alfvén waves arriving directly from Io; (ii) secondary reflected Alfvén wing spots (RAW) resulting from Alfvén wave reflections within the Io plasma torus prior to their escape to high latitudes; (iii) a third kind of spot, named the transhemispheric electron beam (TEB) spot, thought to be generated by electrons accelerated at high latitude in the antiplanetward direction in one hemisphere and hence precipitating in the opposite hemisphere (Bonfond et al., 2008). The lead angle has been measured in the UV as a function of Io's longitude (see Fig. 2b and Bonfond et al., 2009). The MAW lead angle is a consequence of the finite travel time of the Alfvén waves between Io and Jupiter, in particular due to the low Alfvén velocity in the plasma torus. It should thus be modulated by the latitudinal separation between Io and the center of the plasma torus. However, the measured MAW lead angle variations are not consistent with this scheme. This has been interpreted by Bonfond et al. (2009) as a consequence of the poor modeling of the azimuthal component of the magnetic field in the Jovian magnetic field models used. This is supported by the fact that the difference in lead angle between different spots are consistent with the Alfvén wing theory (Fig. 2c), i.e the absolute lead angle value is not accurate, but relative ones are.

In the present paper a set of radio observations are modeled in order to obtain a statistical measurement of the parameters 
a

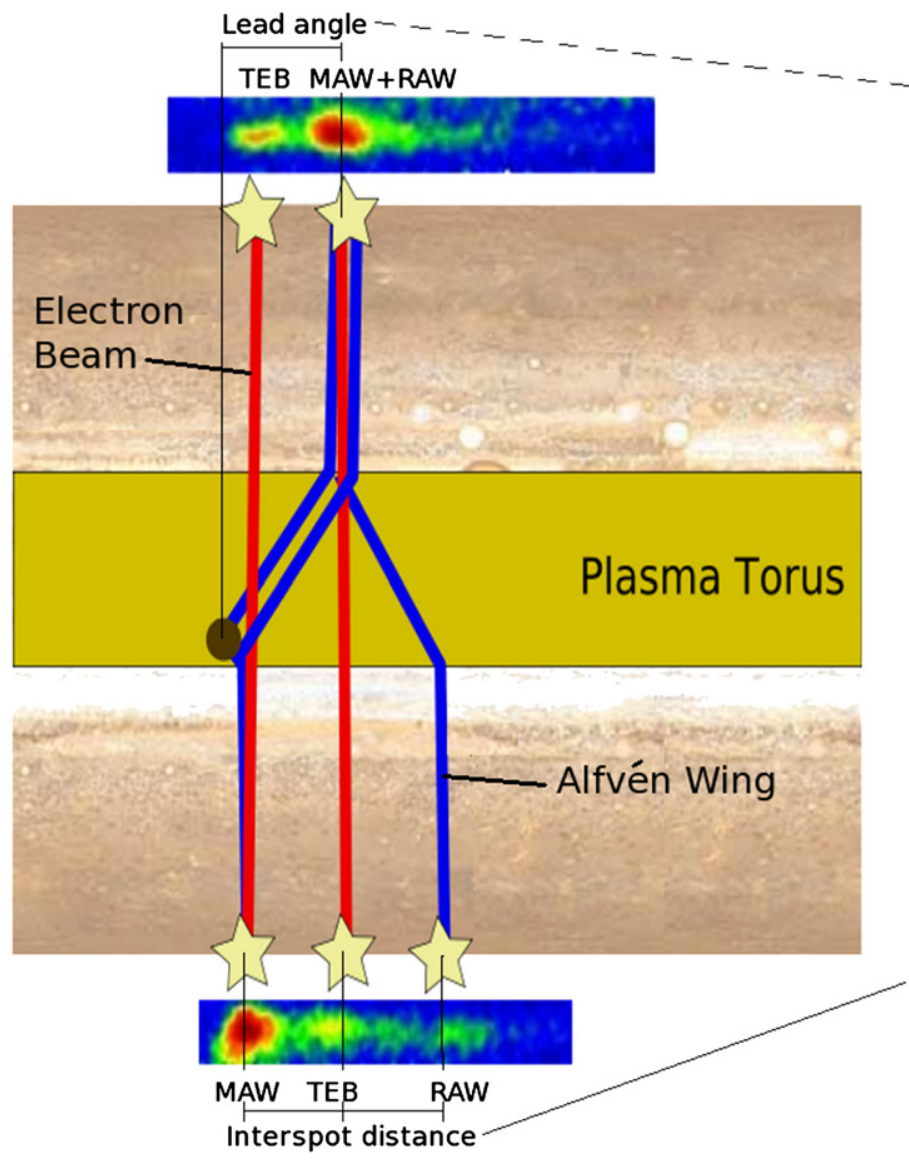

b

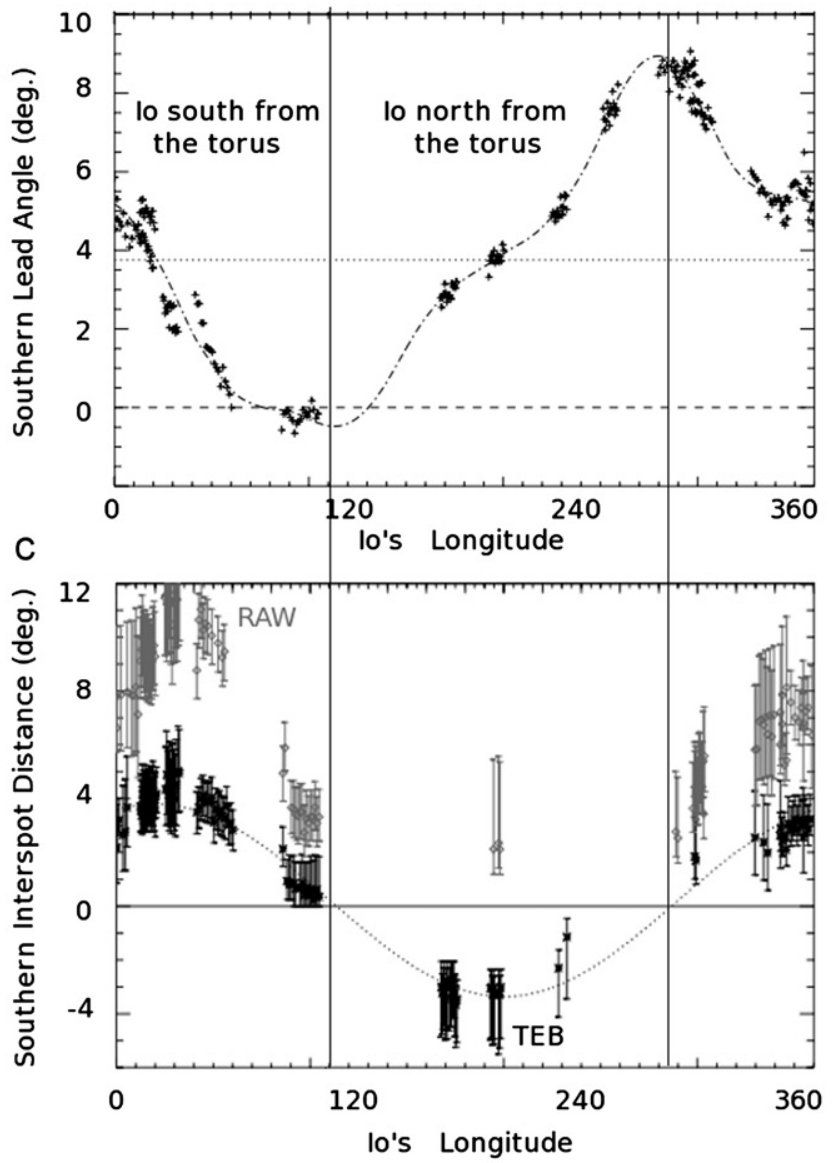

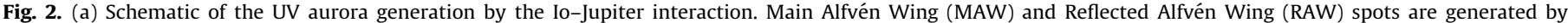

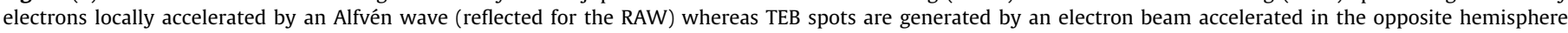

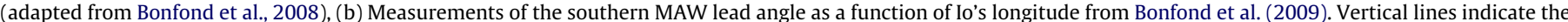

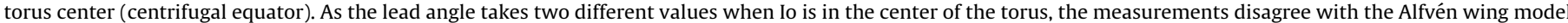

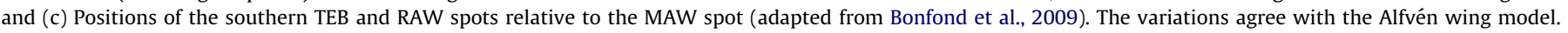

controlling the radio arc shapes: the lead angle of the magnetic field line active in radio, and the velocity of the emitting electrons which defines the theoretical beaming angle profile, as proposed by Hess et al. (2008a). We use the simulation code introduced by Hess et al. (2008a) to model ground-based observations made with the Nançay decameter array (France). The Earth's ionospheric cutoff restricts these observations to frequencies higher than $10 \mathrm{MHz}$, so that we do not have to include the corrections for refraction proposed by Ray and Hess (2008). The arc modeling method is presented in Section 2. Confrontation of model results to data is presented in Section 3. Implications are discussed in Section 4 .

\section{Radio arc modeling}

\subsection{Simulation code}

The exoplanetary and planetary radio emission simulator (ExPRES) code computes the visibility of planetary radiosources by a given observer taking into account the 3D geometry of the observation, and making various assumptions on the characteristics of the emission. A detailed description of this code is available in Hess et al. (2008a). Fig. 3a shows a sketch of the geometry corresponding to Io-Jupiter radio arc observations. ExPRES computes the angle between the observer's line of sight and the direction of the radio emission generated by the CMI for any specified distribution of the electrons. The emission is assumed to be produced at the local cyclotron frequency $f_{c e}=\omega_{c} / 2 \pi$, i.e. the frequency of the emission depends on the position of the source along the active magnetic field line, which is defined by the Jovian magnetic field model chosen. The computation is performed for several frequencies (i.e. altitudes) and times, so that the code produces a modeled dynamic spectrum of what should be observed for any given observation configuration, which is directly comparable to the observed dynamic spectra.

EXPRES proceeds in two steps. It first computes for each time step the angles between the observer's line of sight and the magnetic field vectors along the flux tube active in radio. This active magnetic flux tube is assumed to be fixed in Io's frame, with a constant shift in longitude at the equator $\delta \lambda$. This means that the flux tube rotates around Jupiter along with Io. But the topology of the tube and the position of its ionospheric footprints relative to those of the instantaneous IFT depend on its longitude in the Jovian frame. The motion and the deformation of the active flux tube with respect to its longitude is computed by the code using a given magnetic field model.

Here we used the VIP4 Jovian magnetic field model to compute the topology and the magnetic field intensity along the active flux tube. VIP4 is a fourth order multipolar model of the Jovian internal magnetic field derived from infrared (IR) observations of 
a

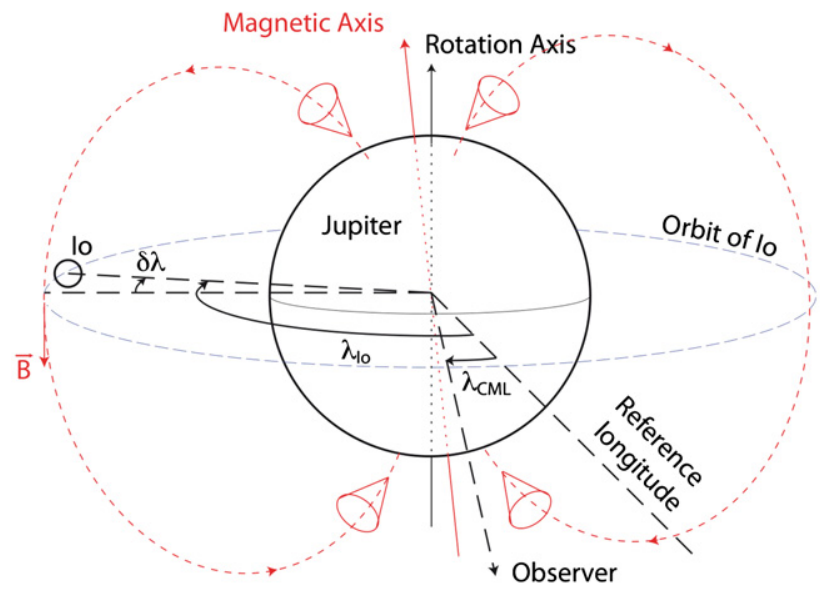

b

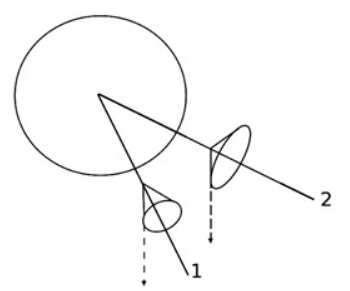

observer

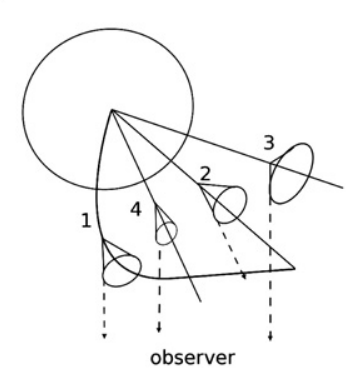

Fig. 3. Sketch of the geometry of radio arc observations (a) and geometrical issues affecting the fitting of the modeled arcs: (b) a modification of the lead angle can be balanced by a modification of the beaming angle (and hence the of the electron velocity). (c) a magnetic field line bending (1) not modeled by the magnetic field model (2) can be balanced by a modification of the beaming angle (velocity) and/ or the lead angle (3-4).

the positions of the Io footprints combined with Voyager and Pioneer magnetic filed measurements (Connerney et al., 1998). It is the most accurate published model of the magnetic field geometry for field lines crossing Io's orbit.

Once the angle between the line of sight and the magnetic field direction is obtained by a scalar product at each frequency, we compute the beaming angle for each source along the active flux tube. This angle is deduced from the cyclotron maser theory assuming a specific unstable electron distribution for amplifying the radio waves. Here we use a loss-cone distribution, because former studies showed that the corresponding theoretical beaming angle profile $\theta(f)$ leads to the most accurate modeling of Jovian radio arcs (see discussions in Hess et al., 2008a; Ray and Hess, 2008):

$\theta=\arccos \left(\frac{v}{c N \cos \alpha}\right)=\arccos \left(\frac{v}{c N} \frac{1}{\sqrt{1-\omega_{c} / \omega_{\text {surf }}}}\right)$

where $\omega_{\text {surf }}$ is the electron cyclotron frequency at the top of the Jovian ionosphere, $\alpha$ is the loss-cone opening angle and $v$ the velocity of emitting electrons. In the present paper we assume a refraction index $N=1$, consistent with the results of Ray and Hess (2008) for sources emitting at frequencies larger than $10 \mathrm{MHz}$. At lower frequency, the refraction index decreases and causes the beaming angle to decrease as well. This effect has been modeled by Ray and Hess (2008) for Io-controlled emissions and may explain a similar trend of the beaming angle of the non-Io emissions observed by Imai et al. (2008). The dependence of the loss-cone opening angle and of the beaming angle on the electron cyclotron frequency and on the emitting electron velocity, in the $N=1$ approximation, is displayed in Fig. 4. Finally, if the beaming

angle and the angle between the line of sight and the magnetic field vector in the source differ by less than $1^{\circ}$ (hollow cone thickness), we conclude that the source is visible, so that radio emission appears in the simulated dynamic spectrum. This value of the hollow cone thickness was suggested by various observations (Queinnec and Zarka, 1998; Kaiser et al., 2000).

\subsection{Fitting method}

The time and frequency coordinates along each observed arc were first measured manually. From this first approximation, an automated routine finds the line of maximum intensity along the arc in the $t-f$ plane. Arc width is then determined by searching, for each pixel of the line of maximum intensity, in the direction locally perpendicular to this line, the levels equal to half the intensity of the corresponding pixel. We obtain by this method a 1 -bit mask of the arc shape in the $t$ - $f$ plane.

This mask is to be compared to simulated ones. For each observed dynamic spectrum we performed about 2000 runs of ExPRES with different values of the resonant electron velocity $v$ (controlling the radio beaming angle) and of the lead angle $\delta \lambda$. Then we cross-correlated the observed mask with the simulated ones. Fig. 5 shows the masks of an observed Io-C arc recorded in Nançay on August 7th, 1999 (in green), superimposed to its best fitting simulated arc (in red). Fig. 6 displays the correlation coefficients obtained from the fit of this Io-C observation with all its simulated counterparts as a function of the electron velocity and of the lead angle. The diagonal appearing on the figure is due to the covariance - of geometrical origin (see Section 2.3.1) - of the parameters $v$ and $\delta \lambda$. As the correlation coefficient decreases abruptly perpendicular to this diagonal, we zoomed into it by performing simulations on a finer grid of lead angles and electrons velocities.

In the cross-correlation of 1-bit masks where the fraction of pixel having the value 1 is small compared to the total number of pixels in each mask, the correlation coefficient is approximately the number of pixels with value 1 in both masks divided by the total number of pixels with value 1 in either mask. A correlation coefficient to 0.5 means that the observed and simulated masks share $\sim 50 \%$ of their pixels with value 1 . More generally, if the fraction of pixels " 1 " in either mask is $a(0 \leq a \leq 1)$, and relative fraction of pixels " 1 " in common is $b(0 \leq b \leq a)$, then the correlation coefficient is $\sim(b-a) /(1-a)$. This tends to prevent correlation coefficients to reach values close to 1 . Another limiting factor comes from the arc width: it is determined empirically in the observations (half-power width around the line of maximum intensity) whereas it is modeled as constant in ExPRES simulations.

\subsection{Model issues}

\subsubsection{Lead angle versus beaming angle}

As two input parameters of ExPRES, the lead angle and the electrons velocity (controlling the radio beaming angle) influence the detection of emission, there may be situations for which non-unique solutions exist. Such a geometry is illustrated on Fig. 3b. Its effect on the uniqueness of the solution is illustrated by the trend of the correlation coefficient versus lead angle and velocity on Fig. 6 . We see that a lead angle modification can be balanced by a beaming angle (hence electron velocity) modification. However, as the magnetic field line topology varies for different lead angles, and as our observations cover a broad range of frequencies-and thus of altitudes and geometries-we can generally find one clear maximum in the correlation coefficient matrices such as Fig. 6, from which an unique solution can be 
a

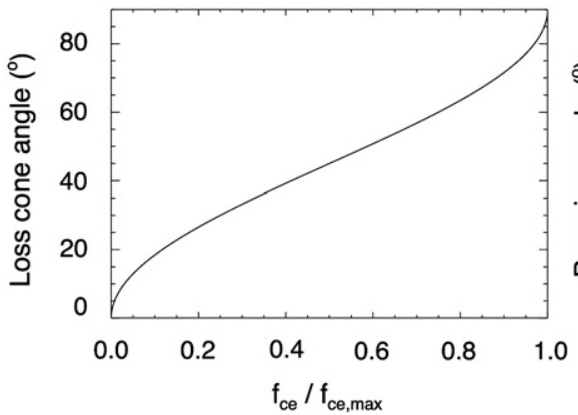

b

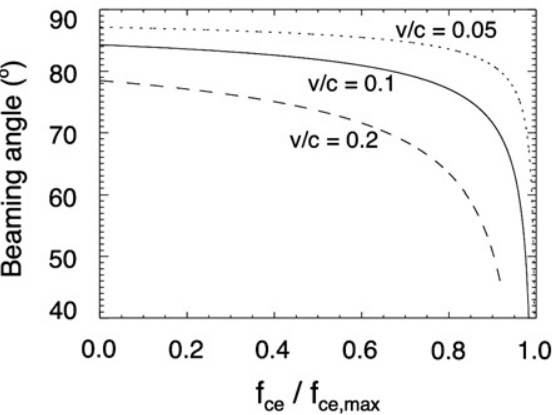

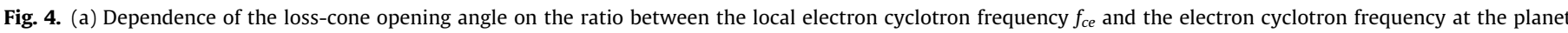

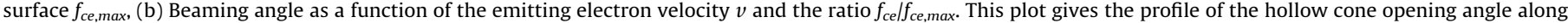
the emitting field line for various electron velocities. The beaming angle decreases with increasing velocity.

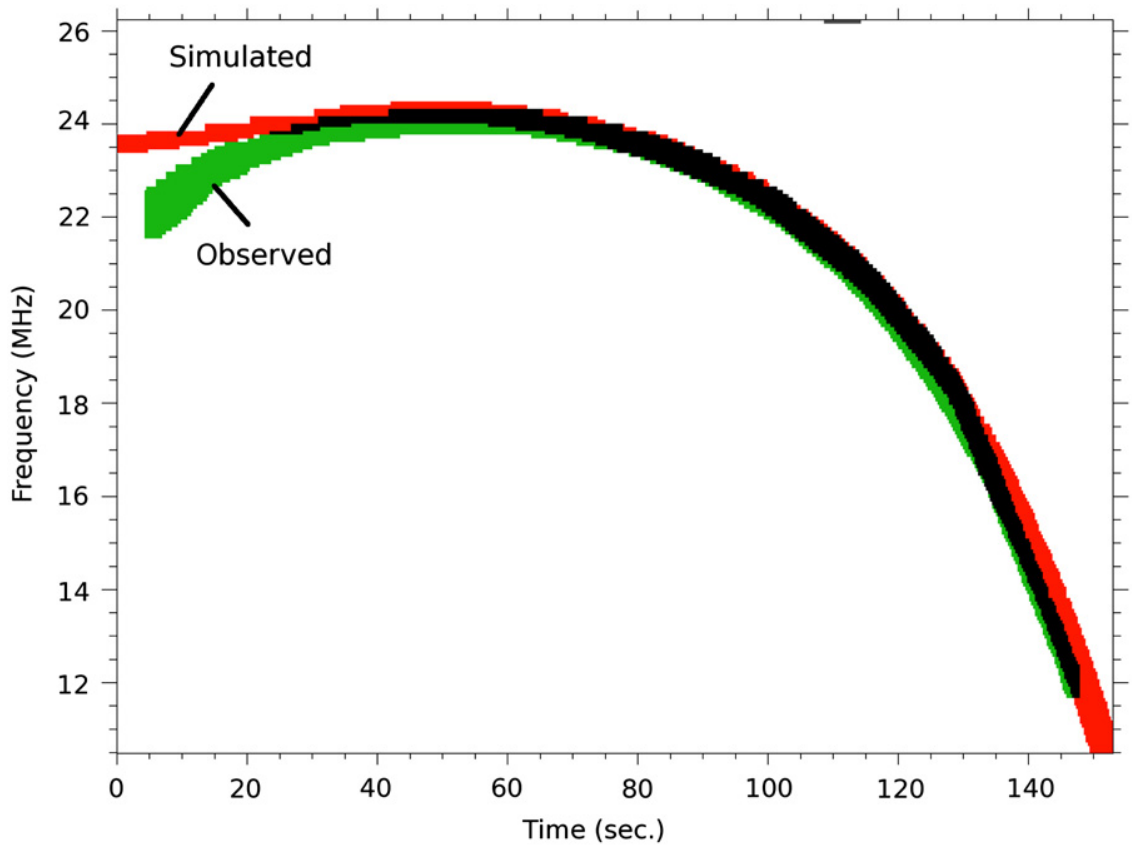

Fig. 5. Dynamic spectrum of a 1-bit mask of the Io-C arc observed on 7 August 1999, and of the fitted simulated arc.

determined. Overall, the covariance between lead angle and electrons velocity introduces an error bar of approximately $\pm 1^{\circ}$ in our measurements of $\delta \lambda$. Note that, due to geometrical (EastWest) symmetry, the Io-B and Io-D diagonals have opposite slopes with respect to the Io- $\mathrm{A}$ and Io-C cases.

\subsubsection{Field line bending, and lead and beaming angles}

The VIP4 magnetic field model was built to match, along Io's orbit, the latitudes of the modeled IFT footprints to observations of IR Io-induced spots in the ionosphere. The VIP4 is much less constrained by the longitude correspondence between the instantaneous spots and Io's position, in particular due to the lead angle caused by low Alfvén velocities in the torus. As a consequence, the azimuthal field component is less well represented, and the field line mapping is poorly constrained in longitude. This constitutes a source of uncertainty in our measurements.

Fig. 3c sketches a "realistic" magnetic field line (1) whose bending is not well-described by the magnetic field model (2). In this case a beaming angle that corresponds to observable emission on field line (1) gives no more observable emission on field line (2). For an Io-C observation (which corresponds to the geometry of Fig. 3c), the emissions can thus be well modeled by a field line with a larger lead angle and a larger beaming angle (lower velocity) to balance it (3), or a field line with lower lead angle and smaller beaming angle (higher velocity) (4). For an Io-D emission the lead angle versus velocity variations that compensate each other to ensure a good fit are opposite to the Io-C case (i.e. a larger lead angle requires a higher velocity), due to East-West symmetry. The lead angle which gives the best correlation with the observation depends on the magnetic field topology of the emitting field line from which emission is detected at the time of the measurements, and on the range of frequencies observed. It may thus vary significantly from one observation to another, causing a spread of the measurements.

\subsubsection{Surface magnetic field}

The available magnetic field models infer the magnetic field strength at high latitudes from spacecraft measurements performed at low latitudes. This can result in a large difference between the modeled and actual magnetic field strengths at high latitudes. The VIP4 model that we use predicts maximum (surface) gyrofrequencies that are lower than the observed one, the discrepancy being larger in the northern hemisphere. We 


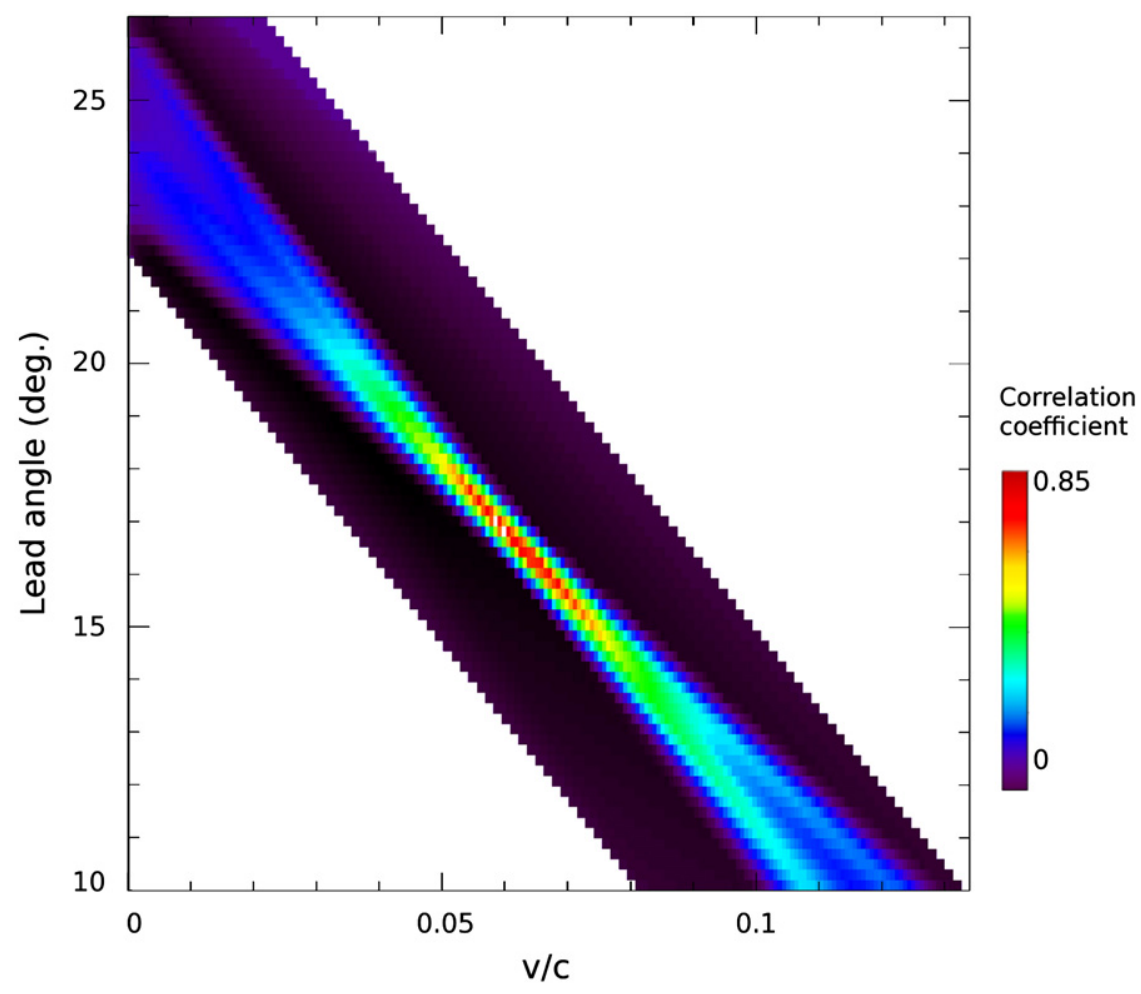

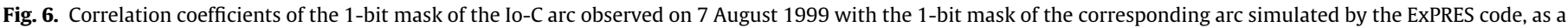

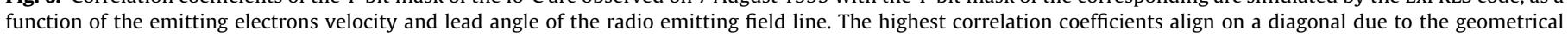
balance of the lead angle and the beaming angle.

attempted to model six dynamic spectra of Io-B emissions: in each case, we obtained a modeled radio arc with a shape very similar to the observed one, but which did not reach frequencies as high as those observed. Although the maximum frequency of the simulated arcs almost reach the surface electron cyclotron frequency predicted by the VIP4 model for the emitting field line, this frequency was still a few $\mathrm{MHz}$ below the observed one. This was already the case in Hess et al. (2008a). Grodent et al. (2008) proposed a modification of the VIP4 model in the northern hemisphere in order to reproduce more accurately the Io, Europa and Ganymede footpaths. Their perturbed model suggests that the the surface magnetic field strength could be larger than predicted by the VIP4 in our region of interest, but cannot be considered as a self-consistent magnetic field model for computing field lines far above the satellite footpaths. To model northern emissions we thus need a more accurate magnetic model, as will certainly be provided by JUNO measurements.

\section{Results}

\subsection{Parameter determination}

We fitted 32 Io-C arcs and 20 Io-D arcs observed by the Nançay decameter array (http://www.obs-nancay.fr/a_index.htm) between 1991 and 2007 (plus 6 Io-B arcs as mentioned above, but that will not be discussed further below). These arcs were selected for the low level of interference present at the time of the observation, and they are spread over the 1991-2007 period, so that they cover more than a revolution of Jupiter around the Sun (i.e. observations were performed at all the possible latitudes of the Earth in the Jovicentric reference frame).

Fig. 7 summarizes the best fitted electron velocity and lead angle for all modeled arcs. The correlation coefficients between the observations and the simulations range from $\sim 0.3$ up to 0.92 , indicating the good quality of the fits of arc $t-f$ shapes. Parameters for Io-C and Io-D arcs are concentrated along two oblique lines, shown be the dash-dotted lines on the figure. Their slope is different from that of the oblique line observed in the distribution of correlation coefficients for each individual arc (such as Fig. 6), which is caused by the balance between lead angle and beaming angle (electron velocity), and thus has a different meaning. The oblique line in Fig. 7 denotes a relation between the lead angle and the velocity that can be physical or may result from field line bending and its effect on the lead and beaming angles of the detected radio emission, as explained in Section 2.3.2.

\subsection{Variations with Io's longitude}

Fig. 8a shows the measured (model) lead angles versus the mean longitude of Io during the observed emissions. It should be noted that since the spread in Io longitude of the Io-D arcs is largely wider than those of the Io-C arcs, most of the information on the longitudinal variations of the measured parameters are obtained from the Io-D arcs. A modulation is visible in our data, as the minimum (negative) values of the lead angle correspond to a range of Io longitude comprised between $\sim 150^{\circ}$ and $\sim 220^{\circ}$, while the higher (positive) values of the lead angle are grouped between $\sim 270^{\circ}$ and $\sim 100^{\circ}$. In order to emphasize this longitudinal variation, we fitted a cosine variation $\left(a \cos \left(\lambda_{I o}-\phi\right)+b\right)$ to the displayed data. The best fit is obtained for $\phi \sim 0^{\circ}$ and $a \sim 9^{\circ}$ and $b \sim 12^{\circ}$. Varying the weighting of the fitted points, and due to their large scatter, we estimated that the accuracy on the determination of $\phi$ is no better than $\pm 20^{\circ}$. The correlation coefficient of the data with the best fitted cosine curve is $\geq 0.5$.

Fig. $8 \mathrm{~b}$ shows the measured (model) electron velocities versus the same abscissa as Fig. 8a. As for the lead angles, we fitted a 


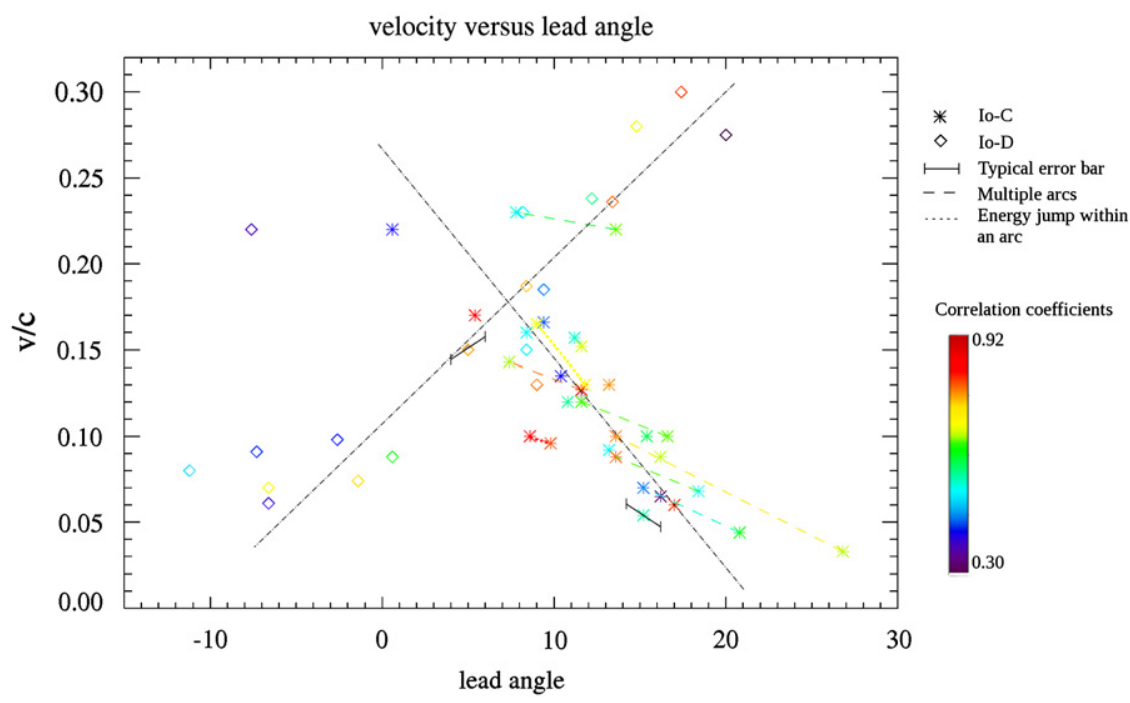

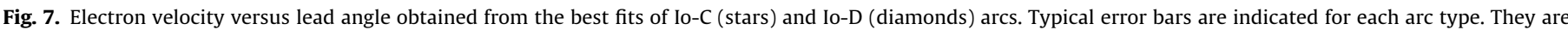

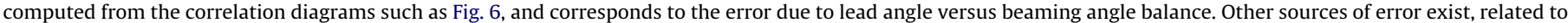

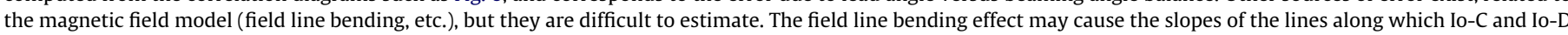

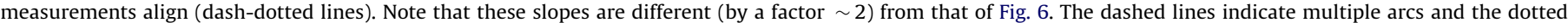
lines arcs revealing velocity and lead angle drops.

a

Io's reference frame

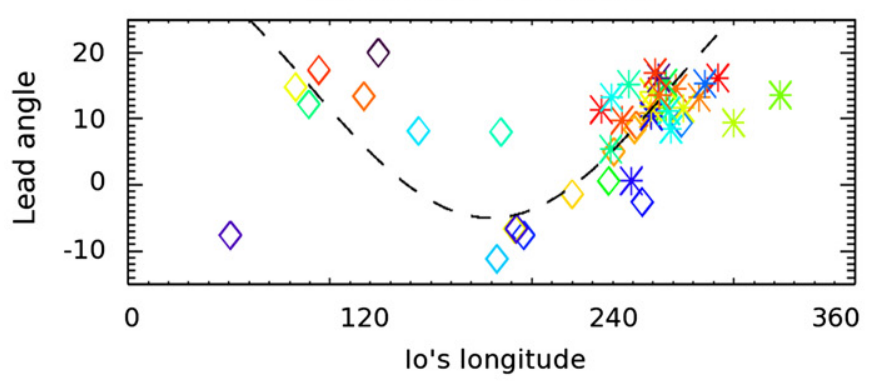

b

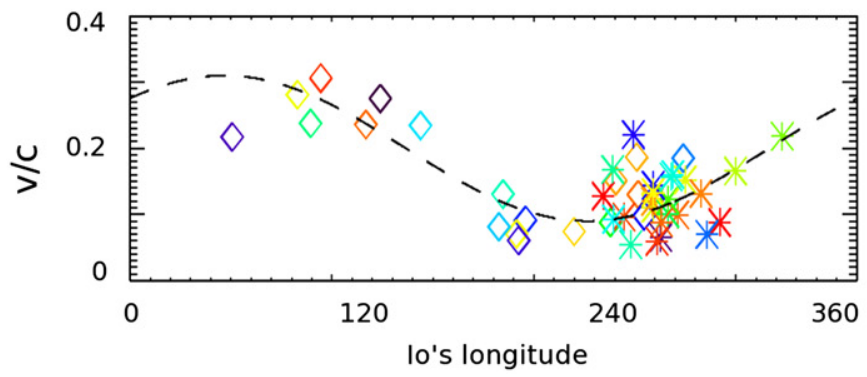

C

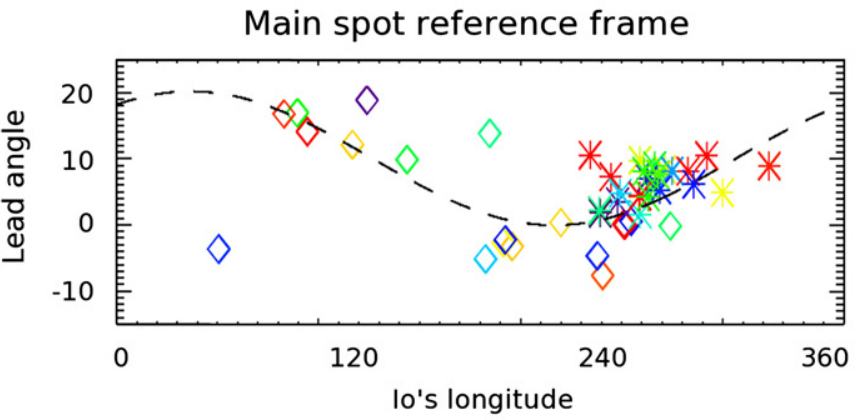

d

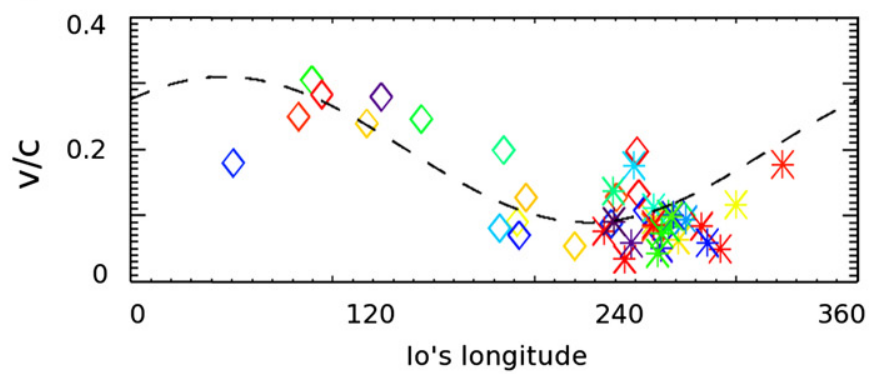

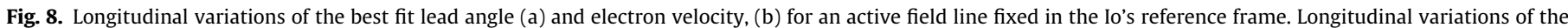

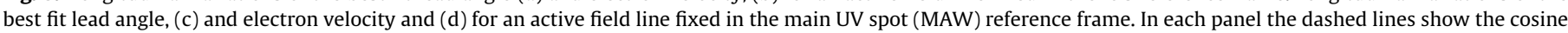

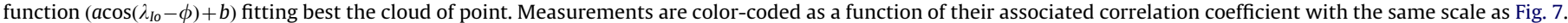

cosine function to the observed velocity variations. The best fit is obtained for $\phi \sim+50^{\circ}$ (with $a \sim 0.1$ and $b \sim 0.2$, and a correlation coefficient up to 0.76 ). We note that there is a phase-shift of about $50^{\circ}$ between the lead angle and electron velocity variations.

The lead angle determination gives negative values of the lead angle in several cases. These negative values cannot correspond to physical lead angles, since it would violate causality (nothing can happen before the magnetic field lines encounter Io). These negative values are most probably caused both by inaccuracies of the magnetic field model mapping, as discussed above, and by uncertainties inherent to our modeling method.
As shown by Bonfond et al. (2009) for the MAW UV spot, and explained above, the lead angles deduced using the VIP4 model are inaccurate close to Jupiter, because the azimuthal component of the magnetic field is poorly modeled with the VIP4, causing large errors close to the planet. This drawback also applies to our radio modeling because the decameter radio sources are located at altitudes $\leq 0.5$ Jovian radius above Jupiter's ionosphere. It is thus very difficult, if at all possible, to interpret the variations of the lead angles with Io's longitude displayed in Fig. 8a. As Bonfond et al. (2009) showed that the difference in lead angle between different UV spots are consistent with the Alfvén wing theory 
(i.e the longitude shifts of the spots relative to each other are well reproduced by the magnetic field), we can bypass the above problem by simulating an active field line fixed in the MAW spot reference frame rather than in Io's one.

New fits were thus computed with an active field line fixed the MAW spot reference frame (it is not enough to shift in longitude the results of previous fits because the MAW spot reference frame is non-linearly related to Io's reference frame-cf. Fig. $2 \mathrm{~b}$ - and the shift between the two varies along the duration of a radio arc). Fig. 8c shows the new measured (model) lead angles versus the mean longitude of Io during the observed emissions. They can now be fitted by a cosine function with a phase about $+40^{\circ}$ (and $a \sim 7^{\circ}$ and $b \sim 10^{\circ}$ ). The correlation coefficient in this case is close to that of the fit of Fig. 8a ( $\geq 0.5$ ). Similarly, Fig. 8d shows the measured (model) electron velocities versus Io's longitude for an active field line fixed in the main spot reference frame. The new phase $\left(\phi \sim+70^{\circ}\right)$ and amplitude $(a \sim 0.1$ and $b \sim 0.2)$ of the cosine fit are close to the values for Fig. $8 b$, and thus quite robust with respect to the chosen reference frame. The corresponding correlation coefficient is slightly increased as compared to Fig. $8 \mathrm{~b}$ (up to 0.83 ). The phase shift between the lead angle and electron velocity variations is reduced to $\sim 30^{\circ}$.

Moreover it should be noted that the modeling leads to a lower number of negative lead angles, the remaining negative values being generally closer to 0 than in the previous modeling. The remaining negative values should still be attributed to inaccuracies in the magnetic field model and in our modeling.

Thus, in the main spot reference frame, the lead angle of the active field line and the velocity of the electrons involved in the radio emission follow similar variations. These variations are also close to that of the TEB and RAW UV spots (Fig. 2b) and to the latitude variations of the torus center relative to Io $\left(\sim+20^{\circ}-\right.$ see Fig. 6 of Queinnec and Zarka, 1998).

We will assume hereafter that main spot reference frame is best adapted to describe the magnetic field line geometry at high latitudes, and all the results discussed further were obtained in that frame.

\subsection{Lead angle and electron velocity statistics}

Fig. 9 shows the histogram of the electron kinetic energy (panel a) and of the lead angles (panel b) corresponding to the modeled arcs. Each arc contribution to the histograms has been

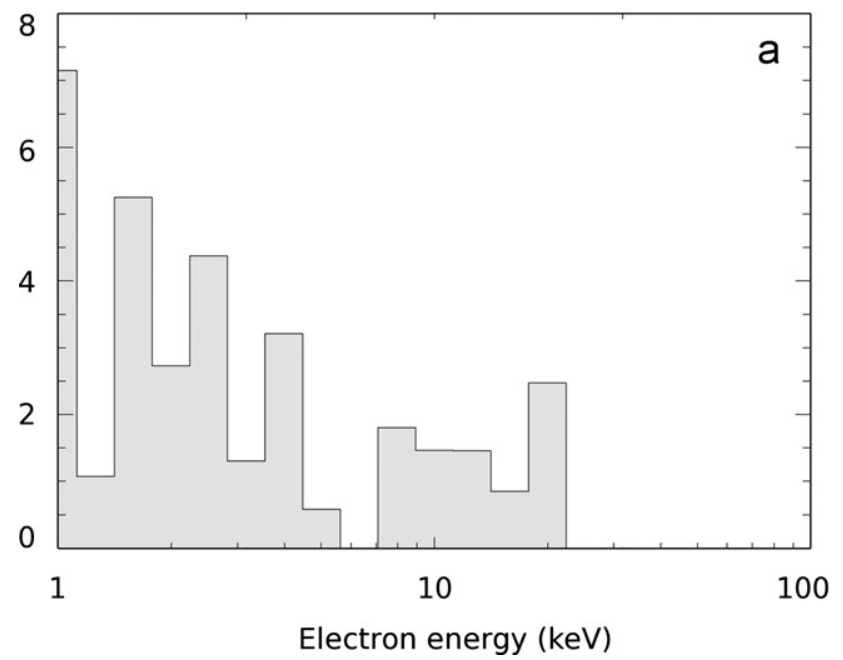

weighted by the correlation coefficient between the observed and modeled arc, so that poorly fitted arcs contribute less to the statistics. The electron energy covers a range between a few keV and $20 \mathrm{keV}$ (with a weighted average about 3-4 keV). These values are in agreement with the measurements of the energy of the electrons emitting the Jovian millisecond bursts made by Zarka et al. (1996), Hess et al. (2007a, 2009). They are also consistent with estimates of the energy of the precipitating electrons in the UV tail prolongating spots generated by the Io-Jupiter interaction (Bonfond et al., 2009). The distribution of lead angles (relative to the MAW UV spot) is mainly comprised between $0^{\circ}$ and $10^{\circ}$, not inconsistent with the average value of $10^{\circ}$ (relative to the IFT) found by Queinnec and Zarka (1998), but much less dependent of the VIP4 model inaccuracies.

\subsection{Multiple arcs}

We observed, in six Io-C dynamic spectra, multiple arcs during the same observation. These cases are indicated by the dashed lines in Fig. 7. Such multiple arcs have been known for a long time and interpreted as the signature of bounces of the Alfvén wave generated by the Io-Jupiter interaction (Queinnec and Zarka, 1998). These bounces also generate multiple spots observed in the UV and the IR. The interspot angular separation observed in the IR is $\sim 5^{\circ}$ (Connerney and Satoh, 2000). UV observations show that the interspot distance may vary with the position of Io relative to the torus center, with an average value of $\sim 6^{\circ}$ (Gérard et al., 2006; Bonfond et al., 2008, 2009, Fig. 2).

We fitted these multiple arcs to obtain the lead angle difference and to evaluate the electron energy variation between them. The histogram of lead angle differences is shown on Fig. 10a. Except in one case (1 February 2000), the lead angle differences are consistent with the $5-6^{\circ}$ values measured in the UV and IR, which confirms the close relationship between multiple radio arcs and the multiple UV spots. Moreover the "anomalous" lead angle difference of 1 February 2000 is equal to $2 \times 6.5^{\circ}$, which could be consistent with our interpretation if we assume that one intermediate arc is missing. Fig. 10b shows the velocity variations between the multiple arcs. It corresponds to a loss of about $0.02 c$ in velocity from one arc to the next. For an averaged velocity of $0.11-0.125 c(3-4 \mathrm{keV})$, this corresponds to an energy loss about $1 \mathrm{keV}$.

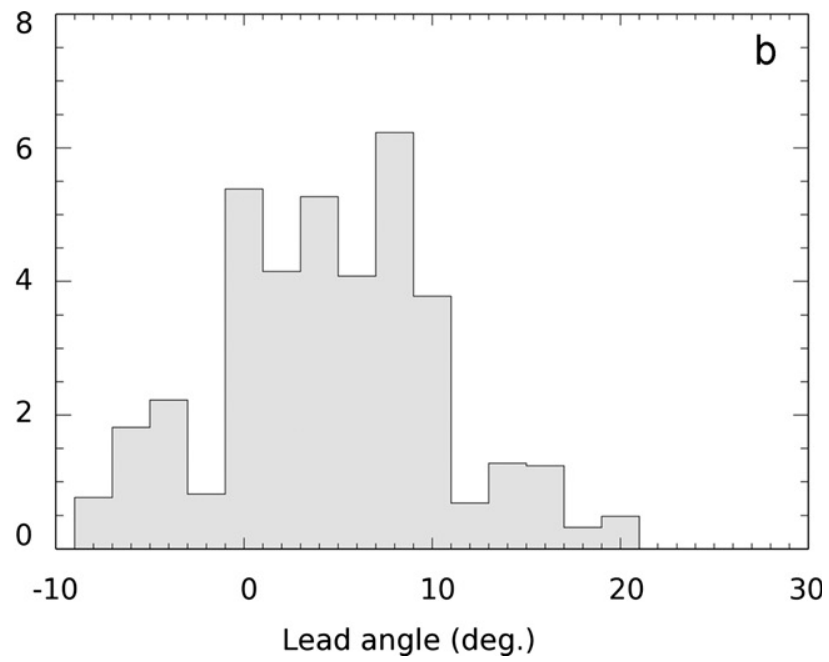

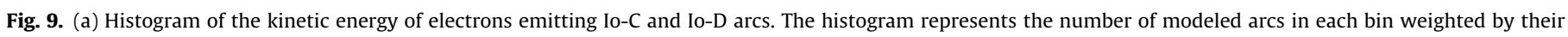

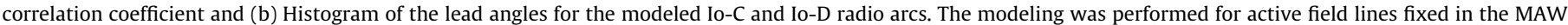
reference frame. 
a

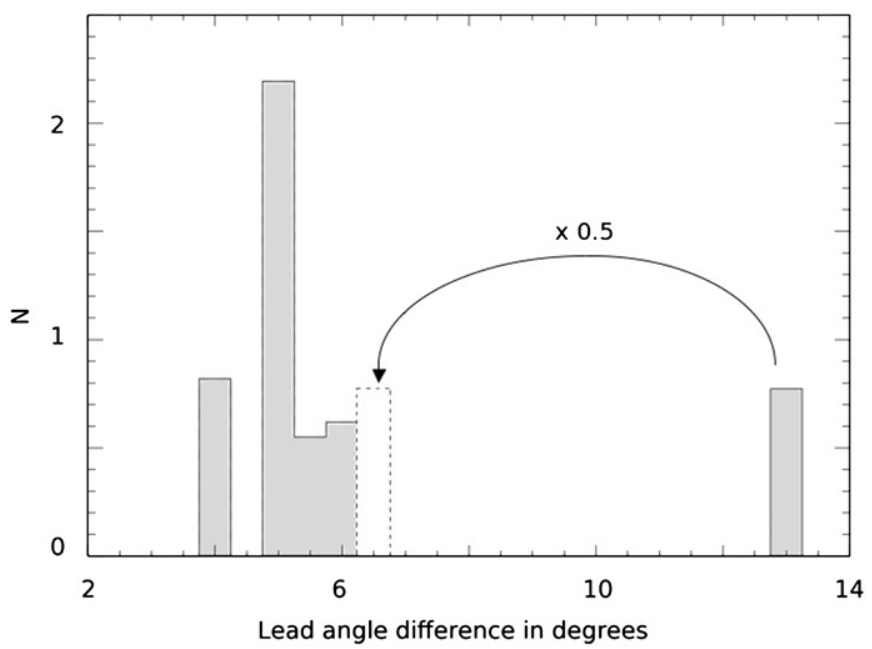

b

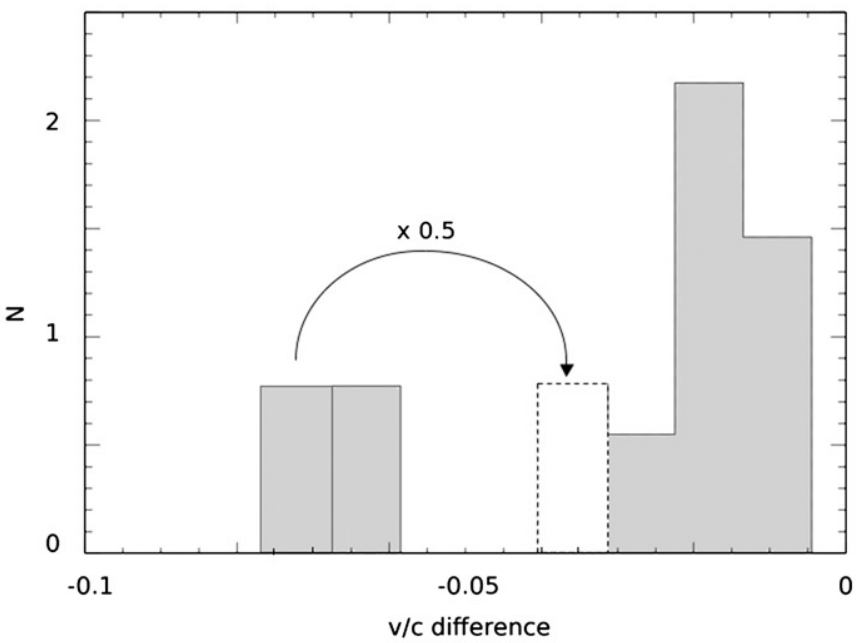

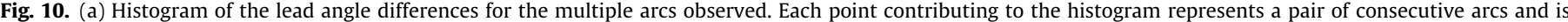

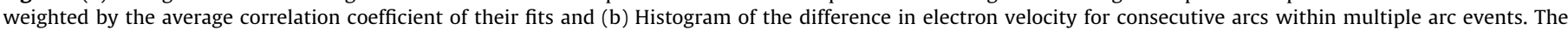

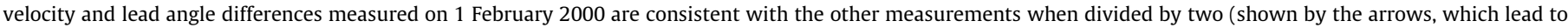
the values indicated by the dashed white bin), as explained in the text.

\subsection{Electron energy drops}

We observed two cases (18 September 1998 and 4 April 2006, both Io-C) for which there is a abrupt change in the arc shape along the arc. This corresponds to energy (velocity) and lead angle drops. We fitted separately the two parts of the arc on either side of the "break", in order to determine the amplitudes of the energy drops, and we found $2.5 \mathrm{keV}$ and $450 \mathrm{eV}$ respectively. These two cases are indicated by the dotted line on Fig. 7. These amplitudes are consistent with the magnetic field aligned potential drops discovered through the analysis of millisecond radio bursts observations (Hess et al., 2007a, 2009). But these observed potential drops were related to moving solitons, not long-lived enough to explain the drops in energy that we observe here along two arcs with durations of several hours. There are yet too few observations to constrain a physical process at the origin of these observed energy drops, which might be the signature of stable, long-lived magnetic field aligned potential drops. The associated lead angle drops $\left(\sim 1^{\circ}-3^{\circ}\right)$ is not explain either, but due to their small amplitudes they may be artifacts due to the limited accuracy of our measurements and modeling of parts of an arc (necessarily lower than for a complete arc).

\section{Discussion}

\subsection{The loss-cone driven CMI hypothesis}

The modeling performed in the present paper assumes that the CMI is loss-cone driven, which does not mean that the unstable electron distribution is a pure loss-cone distribution, but that the most unstable waves resonate with electrons along the edge of the loss cone of the distribution. This can happen with horseshoe or ring distributions (i.e. with the loss cone superimposed to these distributions), which may more accurately represent the distribution of electrons accelerated up to a few tens of $\mathrm{keV}$. Loss-cone driven CMI has been proposed by Hess et al. (2007b) to explain the millisecond radio bursts at Jupiter, although the electron distribution simulated by the authors is a ring distribution. For the ring and horseshoe distributions it has been shown by simulations (Pritchett, 1984), and observations in the terrestrial auroral regions (Louarn et al., 1990; Ergun et al., 2000) that the so-called shell instability characterized by an emission purely perpendicular to the magnetic field is the most unstable mode. But the shell instability amplifies waves at a frequency slightly lower than the cutoff frequency of the wave (R-X) mode in cold plasmas.

For the CMI to occur, the plasma/cyclotron frequency ratio $\left(\omega_{p} / \omega_{c}\right)$ has to be lower than $\sim 0.1$. The emissions at Earth, either shell or loss-cone driven, can only occur in density cavities due to the relatively large $\omega_{p} / \omega_{c}$ ratio in the auroral region. These cavities are caused by the plasma acceleration by strong electric potential drops, which deplete the cold plasma component, only allowing accelerated plasma, with energies of few $\mathrm{keV}$, inside the cavity. The hot plasma allows electrons to emit at frequencies lower than the cold plasma cutoff, down to a frequency close to the relativistic electron cyclotron frequency $\left(\sim \omega_{c}\left(1-v_{t h}^{2} / 2 c^{2}\right)\right.$, with $v_{t h}$ the mean velocity of the electrons in the hot plasma). Hence at Earth, where the emissions can only occur inside the cavity due to the large $\omega_{p} / \omega_{c}$ ratio everywhere else, the emissions are always shell-driven (perpendicular) since it is the most unstable mode in a hot plasma. However the efficiency of the shell-driven CMI quickly decreases when the cold component of the plasma becomes not negligible (Pritchett, 1984). The losscone driven $\mathrm{CMI}$ is then the process by which the radio waves are dominantly emitted in a cold plasma. At Jupiter the $\omega_{p} / \omega_{c}$ ratio is low everywhere, so that loss-cone driven emissions (above the cold plasma cutoff) can be emitted everywhere, whereas the shell emissions (below the cold plasma cutoff) can only be emitted in hot plasma cavities.

Since a wave generated by a shell-driven instability must be emitted in a cavity, it should experience a strong refraction on the cavity boundaries. This refraction should be stronger for the higher densities near the planet. Ultimately the shell driven instability could thus emit waves with a radio beaming angle profile qualitatively similar to that of loss-cone driven emission. However in that case the refraction should be larger for the longitudes for which the $\omega_{p} / \omega_{c}$ ratio is larger, corresponding to lower magnetic field intensities. When modeled by a loss-cone beaming angle profile, this larger refraction would result in the measurement of larger electron velocities at these longitudes (cf. Fig. 4). An anti-correlation between the surface magnetic field 
strength and the measured (model) electron velocity should then be observed. It is not the case, so the loss-cone driven instability is the most probable instability for the generation of the Iocontrolled Jovian radio arcs.

\subsection{Longitudinal variations}

Both the lead angles and the velocities of the emitting electrons vary with Io's longitude following a sinusoidal curve. The lead angle relative to the UV main spot is best fitted with a cosine function $\left(\cos \left(\lambda_{I o}-\phi\right)\right)$ with a phase-shift $\phi \sim 40^{\circ}$, whereas the electron energy is phase-shifted by $\phi \sim 70^{\circ}$. We estimated that the accuracy on the determination of $\phi$ is no better than $\pm 20^{\circ}$. Io's latitude relative to the torus center varies as a cosine function with a phase shift $\phi \sim 20^{\circ}$, the intersections of the equatorial and centrifugal planes corresponding to the longitudes $\sim 110^{\circ}$ and $\sim 290^{\circ}$. Thus, the lead angle of the radio emitting field line relative to the MAW spot can be considered as varying in phase with Io's latitude relative to its torus. The electron velocities follow a similar variation, with a shift about $30^{\circ}$ relative to the lead angles, that might be due to errors on the modeling, on the magnetic field model used, and to the limited accuracy of the cosine fits.

The lead angle of the radio emitting field line relative to the MAW spot is found to vary between $\sim 0^{\circ}$, when Io is at its northernmost position relative to the torus, and $\sim 20^{\circ}$ at Io's southernmost position. Due to the limited accuracy of our modeling, both the absolute value of the lead angles and the amplitude of the lead angle variation are subject to caution. Nevertheless this amplitude (more than $20^{\circ}$ between the highest and lowest values) largely exceeds the uncertainty of our modeling: The spread of the measurements on bins of a few tens of degrees corresponds to a statistical error lower than $\sim 5^{\circ}$. Hence, the existence of this variation is not an artifact of our model and corresponds to an actual, physical variation of the lead angle with longitude.

The shape of the lead angle variation is similar to that of the first RAW spot longitude observed in UV (Fig. 2c). The main difference is that the radio arc lead angle can reach $\sim 20^{\circ}$ while the maximum distance between the first RAW and the MAW spots is only $\sim 12$. But the bulk of the distribution of lead angles is comprised between $0^{\circ}$ and $10^{\circ}$, and larger or negative values may be due to the imperfections of our modeling. Consequently, we suggest here that the measured radio lead angle variations reveal that the Io-controlled radio arcs (the main one in the case of multiple ones) are actually related to the first RAW UV spot rather than the MAW spot. This result is surprising, as it has always been assumed in former studies that these radio emissions correspond to the main UV spot. The existence of secondary radio arcs, whose lead angles relative to the main arc are consistent with the bouncing time of the Alfvén wing in the torus, may be related to the following RAW spots.

The absence of radio arc related to the main UV spot could result from a too efficient parallel acceleration above the MAW spot, leading to the precipitation of most of the electrons to generate the bright UV spot (and subsequent IR emissions), while too few electrons are reflected to generate a bright radio arc. An anti-correlation between the observed IR and radio decameter brightness has been established by Connerney et al. (1993), invoking this mechanism. No systematic correlation study exists for UV and radio emissions. Electron acceleration would be more moderate above the RAW spots, leading to a distribution more favourable to radio wave generation, especially via losscone driven $\mathrm{CMI}$.

The electron velocity variations versus longitude deduced of Io-C and -D arcs modeling indicate that the southern IFT electrons are accelerated at higher velocities when Io is near the southern edge of the torus than when Io is near its northern edge. This may be a consequence of the loss of power of the Alfvén wing when it crosses the torus from edge to edge. Conversely, the energy of the electron emitting in radio does not seem to be correlated to the precipitated power in UV, which tends to peak when Io is at the equator (Serio and Clarke, 2008).

\section{Conclusion}

The modeling of the visibility of more than 50 southern Io-controlled radio t-f arcs using the ExPRES code showed that:

The loss-cone driven CMI permits to model the southern radio arcs with a high correlation coefficient between the model and the observations. The loss-cone driven CMI does not imply a losscone distribution, but rather can be associated to emissions by electrons at the loss-cone border of ring or horseshoe distributions (Hess et al., 2007b). Northern arcs cannot be well fitted, although modeled shapes agree with observed ones, because the VIP4 magnetic field model does not reach a high enough surface gyrofrequency in the northern hemisphere.

The lead angle of the radio emitting field line with respect to the main UV spot resulting from the Io-Jupiter interaction is mainly comprised between $0^{\circ}$ and $10^{\circ}$. This value is somewhat lower than is previous case studies (Lecacheux et al., 1998; Queinnec and Zarka, 1998), much less dependent of the VIP4 model inaccuracies, and in good agreement with Alfvén wave propagation between Io and Jupiter.

The electrons energy is about a few $\mathrm{keV}$, and in some rare cases up to $20 \mathrm{keV}$. This range is consistent with both the energies deduced from the millisecond burst drift rates (Zarka et al., 1996; Hess et al., 2007a, 2009), and with the energy of the electrons precipitating in the UV tail of the Io footprint (Bonfond et al., 2009).

Energy and lead angle drops are observed during two radio events, with amplitudes of resp. $450 \mathrm{eV}$ and $2.5 \mathrm{keV}$. They raise the question of the existence of stable, long-lived magnetic field aligned potential drops.

Multiple arcs were observed in 6 dynamic spectra, from which a regular decrease of the lead angle and electron velocity was measured. The lead angle drop from one arc to the next is consistent with the $5-6^{\circ}$ interspot separation in the UV and IR, for all but one case observed in radio. In the latter case, the values of both the lead angle and the electron velocity drop are twice the typical value, still consistent with IR and UV observations provided that one radio arc in the series is "missed".

Longitudinal variations of both the velocity and the lead angle, computed using a reference at the MAW UV spot (much less dependent of magnetic field model inaccuracies, especially of the azimuthal component), are approximately in phase with the latitude of Io relative to its torus, and with the first RAW spot separation from the MAW spot. This suggest in particular that the (main) radio arcs may be related to the first UV Reflected Alfvén Wing spot rather than to the main UV spot.

The accuracy of our results will be considerably improved when a more reliable Jovian magnetic field model will be available, e.g. after the JUNO mission.

\section{Acknowledgments}

S. Hess thanks F. Bagenal, P. Delamere and V. Dols from the University of Colorado for fruitful discussions. This work was supported by the NASA/GEOSPACE NNX07AF30G grants. We thank L. Denis for running and maintaining the Nançay 
Decameter Array, source of the data used in this paper. The Nanç ay Radioastronomy Station from the Paris Observatory is associated to the French Centre National de la Recherche Scientifique (CNRS) as Unité de Service et de Recherche No. 704, and it also acknowledges the support from the Conseil Régional de la Région Centre in France. Bertrand Bonfond was supported by the PRODEX program managed by ESA in collaboration with the Belgian Federal Science Policy Office.

\section{References}

Bagenal, F., 1994. Empirical model of the Io plasma torus: voyager measurements. J. Geophys. Res. 99, 11043-11062.

Bonfond, B., Grodent, D., Gérard, J.-C., Radioti, A., Dols, V., Delamere, P.A., Clarke, J.T., 2009. The Io UV footprint: location, inter-spot distances and tail vertical extent. J. Geophys. Res. Space Phys. 114 (A13) 7224-+.

Bonfond, B., Grodent, D., Gérard, J.-C., Radioti, A., Saur, J., Jacobsen, S., 2008. UV Io footprint leading spot: a key feature for understanding the UV Io footprint multiplicity? Geophys. Res. Lett. 35 5107-+

Carr, T.D., Desch, M.D., Alexander, J.K., 1983. Phenomenology of magnetospheric radio emissions. Phys. Jovian Magnetosphere, 226-284.

Connerney, J.E.P., Acuña, M.H., Ness, N.F., Satoh, T., 1998. New models of Jupiter's magnetic field constrained by the Io flux tube footprint. J. Geophys. Res. 103 (12), 11929-11940

Connerney, J.E.P., Baron, R., Satoh, T., Owen, T., 1993. Images of excited $\mathrm{H}_{3}^{+}$at the foot of the Io flux tube in Jupiter's atmosphere. Science 262, 1035-1038.

Connerney, J.E.P., Satoh, T., 2000. The $\mathrm{H}_{3}^{+}$ion: a remote diagnostic of the Jovian magneto sphere. In: Astronomy, Physics and Chemistry of $\mathrm{H}_{3}^{+}$. Royal Society of London Philosophical Transactions Series A, vol. 358, pp. 2471-+.

Delamere, P.A., Bagenal, F., Ergun, R., Su, Y.-J., 2003. Momentum transfer between the Io plasma wake and Jupiter's ionosphere. J. Geophys. Res. Space Phys. 108 (A6) $11-1$.

Ellis, G.R.A., 1965. The decametric radio emission of Jupiter. Radio Sci. 69D, 1513-1530.

Ergun, R.E., Carlson, C.W., McFadden, J.P., Delory, G.T., Strangeway, R.J., Pritchett, P.L., 2000. Electron-cyclotron maser driven by charged-particle acceleration from magnetic field-aligned electric fields. Astrophys. J. 538, 456-466.

Gérard, J.-C., Saglam, A., Grodent, D., Clarke, J.T., 2006. Morphology of the ultraviolet Io footprint emission and its control by Io's location. J. Geophys. Res. Space Phys. 111 (A10) 4202-+.

Goldreich, P., Lynden-Bell, D., 1969. Io, a Jovian unipolar inductor. Astrophys. J. 156, 59-78.

Goldstein, M.L., Thieman, J.R., 1981a. The formation of arcs in the dynamic spectra of Jovian decameter bursts. J. Geophys. Res. 86, 8569-8578.

Goldstein, M.L., Thieman, J.R., 1981b. The formation of arcs in the dynamic spectra of Jovian decameter bursts. J. Geophys. Res. 86, 8569-8578.

Grodent, D., Bonfond, B., Gérard, J.-C., Radioti, A., Gustin, J., Clarke, J.T., Nichols, J., Connerney, J.E.P., 2008. Auroral evidence of a localized magnetic anomaly in Jupiter's northern hemisphere. J. Geophys. Res. Space Phys. 113 (A12) $9201-+$
Hess, S., Cecconi, B., Zarka, P., 2008a. Modeling of Io-Jupiter decameter arcs emission beaming and energy source. Geophys. Res. Lett. 35, 13107 +

Hess, S., Mottez, F., Zarka, P., 2007b. Jovian S-bursts generation by Alfvén waves. J. Geophys. Res. 112, A11212.

Hess, S., Mottez, F., Zarka, P., 2008b. Generation of the Jovian radio decametric arcs from the Io flux tube. J. Geophys. Res. 113, A03209.

Hess, S., Zarka, P., Mottez, F., 2007a. Io-Jupiter interaction, millisecond bursts and field-aligned potentials. Planet. Space Sci. 55, 89-99.

Hess, S., Zarka, P., Mottez, F., Ryabov, V., 2009. Electric potential jumps in the Io-Jupiter flux tube. Planet. Space Sci. 57 (1), 23-33.

Imai, M., Imai, K., Higgins, C.A., Thieman, J.R., 2008. Angular beaming model of Jupiter's decametric radio emissions based on Cassini RPWS data analysis. Geophys. Res. Lett. 35 17103-+.

Kaiser, M.L., Zarka, P., Kurth, W.S., Hospodarsky, G.B., Gurnett, D.A., 2000. Cassin and Wind stereoscopic observations of Jovian nonthermal radio emissions: Measurement of beam widths. J. Geophys. Res. 105, 16053-16062.

Lecacheux, A., Boudjada, M.Y., Rucker, H.O., Bougeret, J.L., Manning, R., Kaiser, M.L. 1998. Jovian decameter emissions observed by the Wind/WAVES radioastronomy experiment. Astron. Astrophys. 329, 776-784.

Louarn, P. Roux, A, de Feraudy, H., Le Queau, D., Andre, M., 1990. Trapped electrons as a free energy source for the auroral kilometric radiation. J. Geophys. Res. 95, 5983-5995.

Moncuquet, M., Bagenal, F., Meyer-Vernet, N., 2002. Latitudinal structure of outer Io plasma torus. J. Geophys. Res. Space Phys. 107 (A9) 24-1.

Neubauer, F.M., 1980. Nonlinear standing Alfven wave current system at Io-theory. J. Geophys. Res. 85 (14), 1171-1178.

Prangé, R., Rego, D., Southwood, D., Zarka, P., Miller, S., Ip, W., 1996. Rapid energy dissipation and variability of the Io-Jupiter electrodynamic circuit. Nature 379 , 323-325.

Pritchett, P.L., 1984. Relativistic dispersion, the cyclotron maser instability, and auroral kilometric radiation. J. Geophys. Res. 89, 8957-8970.

Queinnec, J., Zarka, P., 1998. Io-controlled decameter arcs and Io-Jupiter interaction. J. Geophys. Res. 103 (12), 26649-26666.

Ray, L.C., Hess, S., 2008. Modelling the Io-related DAM emission by modifying the beaming angle. J. Geophys. Res. 113, A11218.

Saur, J., 2004. A model of Io's local electric field for a combined Alfvénic and unipolar inductor far-field coupling. J. Geophys. Res. Space Phys. 109 (A18) $1210-+$

Saur, J., Neubauer, F.M., Connerney, J.E.P., Zarka, P., Kivelson, M.G., 2004. Plasma interaction of Io with its plasma. In: Jupiter. The Planet, Satellites Magnetosphere, pp. 537-560.

Serio, A.W., Clarke, J.T., 2008. The variation of Io's auroral footprint brightness with the location of Io in the plasma torus. Icarus 197, 368-374.

Wu, C.S., 1985. Kinetic cyclotron and synchrotron maser instabilities-radio emission processes by direct amplification of radiation. Space Sci. Rev. 41, 215-298.

Wu, C.S., Lee, L.C., 1979. A theory of the terrestrial kilometric radiation. Astrophys. J. 230, 621-626.

Zarka, P., 1988. Beaming of planetary radio emissions. In: Rucker, H.O., Bauer, S.J., Pedersen, B.M. (Eds.), Planetary Radio Emissions II. pp. 327-342.

Zarka, P., 1998. Auroral radio emissions at the outer planets: observations and theories. J. Geophys. Res. 103, 20159-20194.

Zarka, P., Farges, T., Ryabov, B.P., Abada-Simon, M., Denis, L., 1996. A scenario for Jovian S-bursts. Geophys. Res. Lett. 23, 125-128. 